\title{
¿Qué tan cuántica es la química cuántica?*
}

\section{How Quantum is Quantum Chemistry?}

\author{
Hernán Accorinti ${ }^{\dagger}$ \\ Juan Camilo Martinez González
}

\begin{abstract}
Resumen
El propósito del presente trabajo será poner de manifiesto que la química cuántica es una disciplina basada primariamente en modelos, y no en teorías que establecen un marco conceptual específico y autónomo. Esta característica permitirá preguntar qué tan cuántica es la química cuántica que se incorpora precisamente a esos modelos. Para ello analizaremos la aproximación Born-Oppenheimer y los modelos de Enlace de Valencia y de Orbital Molecular. Nuestra intuición es que, en la medida en que sea necesario mantener la noción clásica de estructura molecular para dar cuenta de los comportamientos de las sustancias, la química cuántica adquiere, como propiedad distintiva, un carácter híbrido entre la mecánica clásica y la mecánica cuántica.
\end{abstract}

Palabras clave: química cuántica - aproximación Born-Oppenheimer - enlace de valencia y orbital molecular - modelos autónomos

\begin{abstract}
The purpose of this paper is to show that quantum chemistry is a discipline based primarily on models, and not on theories that establish a specific and autonomous conceptual framework. This feature will allow us to question how quantum is the quantum chemistry incorporated in those models. For this purpose, we will analyze the BornOppenheimer approximation and the Valence Bond and Molecular Orbital models. Our intuition is that, to the extent that maintaining the classical notion of molecular structure is necessary to account for the behavior of substances, quantum chemistry acquires, as a distinctive property, a hybrid character between classical mechanics and quantum mechanics.
\end{abstract}

Keywords: quantum chemistry - Born-Oppenheimer approximation - valence bond and molecular orbital models autonomous models

* Recibido: 3 de junio de 2018. Aceptado con revisiones: 9 de agosto de 2018.

† Universidad de Buenos Aires/Agencia Nacional de Promoción Científica y Tecnológica (FONCyT), Argentina. Para contactar al autor, por favor, escribir a: hernanaccorinti@gmail.com.

* Universidad de Buenos Aires/Consejo Nacional de Investigaciones Científicas y Técnicas (CONICET), Argentina. Para contactar al autor, por favor, escribir a: olimac62@hotmail.com.

Metatheoria 9(2)(2019): 5-18. ISSN 1853-2322. eISSN 1853-2330.

(c) Editorial de la Universidad Nacional de Tres de Febrero. Publicado en la República Argentina. 


\section{Introducción}

La filosofía tradicional de la ciencia ha consagrado a la física como la disciplina paradigmática para pensar las categorías epistemológicas que finalmente se han consolidado durante el siglo XX. Recién en las últimas décadas ha cobrado una relativa notoriedad el estudio de ámbitos como la filosofía especial de la química o de la biología. Paralelamente, en los últimos años, se ha desarrollado un área de análisis muy prolífico tendiente a tratar de comprender el papel que juegan los modelos en el proceso de producción del conocimiento científico. Desde esta perspectiva, la bibliografía especializada se ha comenzado a preguntar qué son los modelos, qué tipo de modelos existen, cuál es su función primordial o qué tipo de relación existe entre los modelos y las teorías (ver, por ejemplo, Suárez 1999, Morgan \& Morrison 1999, Morgan 1999, Morrison 1999, 2015, Knuuttila 2005, Weisberg 2013).

En este contexto, uno de los propósitos del presente trabajo será poner de manifiesto la dinámica de la química cuántica como subdisciplina que se desarrolla primariamente a la luz de un conjunto de modelos y no como una teoría que logra desarrollar un programa propio y autónomo de investigación. En particular, caracterizar a la química cuántica como una disciplina que evoluciona a partir de modelos, y no a partir de una teoría programática, habilitará la pregunta en torno a qué tan cuántica es la química cuántica que se incorpora precisamente a esos modelos. Ciertamente si la química cuántica se constituyera primariamente a partir de una teoría, entonces la pregunta carecería de sentido: siendo cuántica, la química cuántica sería parte de la teoría cuántica. Sin embargo, constituyéndose a partir de sus modelos, sí tiene sentido preguntarse qué tan cuántica es la cuántica que aparece en los modelos de la química cuántica.

En este sentido, el análisis de la aproximación Born-Oppenheimer y los modelos de enlace de valencia y de orbital molecular pondrán en evidencia que los modelos en química cuántica resultan independientes respecto de las teorías ya que involucran marcos teóricos incompatibles. Y es precisamente esta independencia y autonomía de los modelos respecto de las teorías la que nos permitiría afirmar, a contramano de lo que dicta la tradición filosófica, que la capacidad cognoscitiva de la química no se encuentra primariamente cifrada en las teorías.

En vista a dichos objetivos, en la primera sección haremos un breve repaso de las principales dificultades a las que debió enfrentarse la química estructural respecto de la noción de enlace covalente. Ello nos permitirá comprender las razones por las cuales fue necesario introducir el formalismo de la mecánica cuántica para encontrar la energía de los sistemas moleculares y así expandir el conocimiento respecto de las sustancias químicas. En la segunda sección abordaremos dos tipos de aproximaciones que se utilizan en el marco de la química cuántica: el enlace de valencia y el orbital molecular por un lado, y la aproximación Born-Oppenheimer por otro. Por último, en la tercera sección trataremos de responder a la pregunta que se plantea el título del presente trabajo, poniendo en evidencia, como dijimos, que la química cuántica es una disciplina peculiar en cuanto que se constituye esencialmente sobre la base de modelos.

\section{Química cuántica: un poco de historia}

Una buena parte de la química moderna se forjó en la década de 1920. Gracias a las leyes de Dalton y a la estequiometría, ya se habían instituido algunos mecanismos mediante los cuales las sustancias se pueden combinar. Sin embargo, aún no quedaba completamente claro cómo los elementos en una molécula se encontraban unidos. Con el descubrimiento del electrón, la química desarrolló teorías que describían los enlaces en sustancias de tipo polar. Estas teorías generalmente explicaban el enlace de dos átomos diferentes como una transferencia de electrones. Este tipo de uniones químicas son las que actualmente conocemos como uniones iónicas. No obstante, los casos donde se unen átomos iguales (unión homopolar) no podían ser explicados por una mera transferencia electrónica debido a que el 
enfoque de Coulomb para cargas puntuales no permitía explicar la fuerza de atracción de los electrones por átomos de la misma naturaleza.

La primera teoría ampliamente aceptada que intentó dar cuenta del enlace químico covalente (homopolar) fue desarrollada en 1916 por Gilbert N. Lewis en el marco de la química estructural. La teoría de enlace de Lewis, que concebía el enlace en términos de pares electrónicos compartidos, se basaba en dos principios fundamentales: el primero de ellos, denominado "regla del octeto", proponía que los átomos en una molécula estable debían estar rodeados por ocho electrones. Esta regla reflejaba aspectos relevantes de la clasificación periódica: por un lado, los ocho elementos de varios grupos de la tabla, y por el otro, el hecho de que la valencia se corresponde con el número de electrones que el elemento debería ganar o perder para obtener un octeto. El segundo principio sostenía que entre dos núcleos atómicos los electrones se comparten en pares, siendo que estos pares se pueden dividir entre pares de enlace y pares libres. Precisamente los diferentes modos en que los átomos comparten electrones dan cuenta del carácter polar o iónico de los compuestos químicos. La relevancia de esta explicación es que a partir de estas características se pudieron conocer, entre otras cosas, algunas propiedades de las sustancias, como su solubilidad o su electronegatividad.

Lewis desarrolló su teoría gracias a un modelo cúbico del átomo. Este modelo contiene ciertas ideas que hasta el día de hoy continúan siendo sumamente relevantes para el ámbito de la microquímica. Por un lado, la idea de que el núcleo atómico (kernel) contiene un conjunto de cargas positivas y una capa interna de cargas negativas que no juegan ningún papel en el comportamiento químico de las sustancias. Y por el otro, el supuesto de que la capa más externa de esos cubos es donde se disponen los electrones que gobiernan el comportamiento de la actividad química. Según Lewis, la explicación resultaba posible dado que el apareamiento de los electrones tenía una naturaleza estática que no obedecía las leyes de Coulomb. En efecto, Lewis consideraba que "las fuerzas eléctricas entre partículas cargadas [electrones] que están muy cerca no obedecen la ley simple del inverso de los cuadrados, que sí se mantiene a grandes distancias" (Lewis 1916, p. 768).

Precisamente si tenemos en cuenta que las restricciones que impone Lewis a las leyes de Coulomb son incompatibles con la descripción electrostática para cargas puntuales, y consideramos que Lewis nunca intentó brindar una descripción cuantitativa de su modelo, entenderemos por qué su postura nunca fue tomada en serio por la comunidad de los físicos (ver Kragh 1977). En efecto, si bien Lewis distinguió entre los tipos contrarios de enlace químico, polar (iónico) y no polar (covalente), nunca pudo establecer un mecanismo que explicara la dinámica del apareamiento entre los electrones. Fue Alfred Parson (1915), mediante la teoría de los magnetones, quien más cerca estuvo de explicar el mecanismo de apareamiento en términos clásicos. Finalmente, recién con el advenimiento de la mecánica cuántica y su aplicación al ámbito de la química se pudo explicar de un modo consistente la relación entre electrones del enlace químico en la molécula de hidrógeno $\left(\mathrm{H}_{2}\right)$.

La primera aplicación de la mecánica cuántica para explicar la unión química fue desarrollada por Walter Heitler y Fritz London (1927). La importancia de su trabajo radicó en demostrar que la unión química entre dos átomos de hidrógeno únicamente podía ser entendida en términos de las leyes de la mecánica cuántica. En ese contexto, los problemas del átomo de hidrógeno y el ion de la molécula de hidrógeno $\left(\mathrm{H}_{2}{ }^{+}\right)$ya habían sido resueltos y había un interés creciente en la comunidad de físicos por intentar expandir estas soluciones para resolver el problema del enlace en moléculas no polares. El modelo más simple de unión química covalente de átomos iguales es el de la molécula de hidrógeno $\left(\mathrm{H}_{2}\right)$, cuyas soluciones representarían la solución al problema de unión homopolar.

Para comenzar a trabajar, Heitler y London tomaron como base de cálculo dos átomos de hidrógeno separados, que lentamente se acercan el uno al otro. Para postular una función de onda que representara el sistema asumieron que a cada núcleo de hidrógeno le corresponde un electrón. De esta manera, la función de onda $\Psi$ representa que el electrón 1 pertenece al núcleo de hidrógeno " $a$ " y el electrón 2 al núcleo de hidrógeno " $b$ ". Análogamente, la función de onda $\Psi^{\prime}$ corresponde a la posibilidad del intercambio de posiciones de los electrones entre los dos núcleos:

$$
\Psi=C_{1} \Psi_{a}(1) \Psi_{b}(2)
$$




$$
\Psi^{\prime}=C_{2} \Psi_{a}(2) \Psi_{b}(1)
$$

siendo que los $c_{1}$ y $c_{2}$ son los coeficientes por calcular, y las $\Psi$ la función de onda monoelectrónica para átomos hidrogenoides. ${ }^{1}$ Debido a que los electrones son partículas idénticas, la función de onda total del sistema podía ser descrita como una combinación lineal de las funciones de onda hidrogenoides en ambos casos:

$$
\Psi_{\text {total }}=\Psi+\Psi^{\prime}=c_{1} \Psi_{a}(1) \Psi_{b}(2)+c_{2} \Psi_{a}(2) \Psi_{b}(1)
$$

Para calcular los coeficientes $c_{1}$ y $c_{2}$ minimizaron la energía utilizando el teorema variacional: ${ }^{2}$

$$
E=\frac{\int \psi H \psi \partial \tau}{\int \psi^{2} \partial \tau}
$$

y encontraron así dos valores diferentes para la energía del sistema:

$$
E_{1}=2 E_{0}+\frac{C+A}{1+S_{12}} ; E_{2}=2 E_{0}+\frac{C-A}{1-S_{12}}
$$

$\mathrm{S}_{12}$ representa la integral de superposición y representa la medida en la cual las dos funciones de onda se solapan una a la otra $\left(\int \psi_{a} \psi_{b} \delta \tau\right)$. La integral $\mathrm{C}$ es la integral de Coulomb $\left(\int \psi_{a} H \psi_{a} \partial \tau\right)$, y A representa la integral de intercambio $\left(\int \psi_{a} H \psi_{b} \partial \tau\right)$. Tanto $\mathrm{A}$ como $\mathrm{C}$ tienen valores negativos, pero $\mathrm{A}$ es mayor que C. Esto implica que en $E_{1}$ se cumple $c_{1} / c_{2}=1$, y que en $E_{2}$ se cumple $c_{1} / c_{2}=-1$. Al principio Heitler y London no se alentaron demasiado debido a que la integral de Coulomb (C) era demasiado pequeña para dar cuenta de la unión homopolar entre los dos átomos de hidrógeno. No obstante, rápidamente se dieron cuenta que el enlace podía ser entendido gracias a la integral de intercambio (A). Pensado entonces a partir de la integral de intercambio A, y no a partir de las leyes de Coulomb, la energía del enlace covalente podía ser descrita con mayor precisión a partir de las siguientes funciones de onda:

$$
\begin{aligned}
\Psi_{\text {total }} & =1 / \sqrt{2}\left[\Psi_{a}(1) \Psi_{b}(2)+\Psi_{a}(2) \Psi_{b}(1)\right] \\
\Psi_{\text {total }}^{\prime} & =1 / \sqrt{2}\left[\Psi_{a}(1) \Psi_{b}(2)-\Psi_{a}(2) \Psi_{b}(1)\right]
\end{aligned}
$$

Sin embargo, hasta este momento no había sido posible discernir entre estas dos funciones de onda posibles, debido a que en el cálculo el espín de los electrones no se había tomado en consideración. En efecto, las propiedades de simetría que exige el principio de exclusión de Pauli son satisfechos sólo por $\Psi_{\text {total }}$, donde los electrones tienen espines antiparalelos. A su vez, $\Psi_{\text {total }}$ corresponde a la solución de energía $E_{1}$ que, al ser menor que $2 E_{0}$ (la suma de las energías de los dos átomos de hidrógeno separados), representa el caso de atracción. En cambio $\Psi^{\prime}{ }_{\text {total }}$, que es el caso en que los espines de los dos electrones son paralelos, corresponde a la solución de energía $E_{2}$ que, al ser mayor que $2 E_{0}$, representa el caso de repulsión. Recién cuando se consideraron estos factores fue posible comprender que la unión entre los dos átomos de hidrógeno neutros sólo es posible cuando las orientaciones relativas de los espines de los electrones son antiparalelas. En otras palabras, para formar un par electrónico no basta sólo con tener un electrón disponible, sino que además es necesario que esos electrones tengan espines opuestos. Gracias a este trabajo, Heitler y London pudieron calcular de manera detallada la energía de enlace y la distancia internuclear en la molécula de hidrógeno. Por lo tanto, finalmente mediante un efecto netamente cuántico, los autores pudieron brindar una explicación detallada de un enlace químico covalente, aduciendo que éste debe ser concebido en términos de las propiedades de simetría relacionadas con el espín del electrón.

\footnotetext{
1 Se denominan 'sistemas hidrogenoides' a todos los sistemas que contienen un electrón (monoelectrónicos) como, por ejemplo, el ion He del átomo de helio, y el ion $\mathrm{Li}^{2+}$ del átomo de litio.

2 En mecánica cuántica, se conoce como teorema variacional al método para obtener una solución aproximada de la ecuación de Schrödinger correspondiente al estado cuántico de energía más baja o estado fundamental. Esto se logra escogiendo una función de onda $\Psi$ aproximada y ajustando los parámetros para lograr una energía mínima. De este modo, la mejor selección de la función de onda $\Psi$ proporciona un límite superior para obtener una energía mínima.
} 
El resultado de esta aplicación de la mecánica cuántica al enlace químico se considera como uno de los trabajos fundadores de la química cuántica. Heitler y London podrían haber desarrollado un enfoque diferente, en el cual la molécula estuviera mejor definida matemáticamente, pero cuyo modelo tuviera poco sentido físico. Sin embargo, en ese caso habría sido imposible dar cuenta de las energías atractivas. Fue necesario agregar una restricción no trivial para que el resultado se volviera físicamente relevante (Gavroglu \& Simões 2012). En particular, fue el Principio de Exclusión de Pauli el que permitió extraer contenido físico del formalismo matemático: precisamente la función de onda $\Psi_{\text {total }}$, que cumple con el principio, es la que corresponde a la energía mínima, y por lo tanto, representa el caso donde las fuerzas de atracción establecen el enlace entre los dos átomos de hidrógeno. Los términos del formalismo matemático obtuvieron, así, un sentido físico relevante en cuanto a la constitución de la molécula. El enfoque de Heitler y London se convirtió en una explicación sólida del enlace en tanto que también permitió explicar, nuevamente a partir del Principio de Pauli, la ausencia de enlace químico entre dos átomos de helio. El enlace entre dos átomos de helio no es posible porque la función de onda que da cuenta de los requerimientos de simetría de los electrones de dicho elemento es la función de onda de repulsión.

Por lo tanto, para Heitler y London el Principio de Exclusión fue una forma de dar respaldo a las decisiones formales con sentido físico en la molécula de hidrógeno. Como los electrones con espines antiparalelos no son idénticos, el principio de Pauli no aplica sobre ellos; y en consecuencia, podemos legítimamente escoger la solución que es simétrica (Heitler \& London 1927). De este modo Heitler y London habían logrado comprender el concepto de valencia: un enlace químico se forma cuando dos electrones de átomos diferentes se combinan para formar vibraciones simétricas siguiendo los requerimientos del Principio de Exclusión. El espín se convirtió, por tanto, en el indicador más importante de la valencia y la unión química. Gracias a él pudieron explicar el apareamiento electrónico en términos cuánticos, de una forma que no tiene un análogo en la mecánica clásica.

\section{Modelos en química cuántica}

En el apartado anterior hemos presentado un breve recorrido histórico de los orígenes de la química moderna, con el fin de poner en evidencia el hecho de que, producto de las dificultades teóricoempíricas derivadas de las uniones homopolares, en el ámbito de la química se impuso la necesidad de repensar y reformular los conocimientos tradicionales en términos de la mecánica cuántica. Sin embargo, el presente trabajo no tiene una finalidad histórica sino filosófica: el desarrollo histórico, siendo subsidiario al interés netamente epistemológico, será útil para comprender las particularidades metodológicas y procedimentales de la química cuántica. En efecto, la introducción del formalismo de la mecánica cuántica para dar cuenta de la estructura electrónica de una molécula, si bien sumamente prolífica en tanto que permitió recomponer y expandir las afirmaciones sobre las sustancias químicas, no fue, como explicaremos, ni inocuo ni a-problemático. Ello supuso una dificultad conceptual que exige, por un lado, una reinterpretación respecto del modo en que se desarrolla el conocimiento en el ámbito de la química y, por el otro, un análisis específico del modo en que la mecánica cuántica se utiliza en el contexto de la química cuántica.

El tratamiento mecánico-cuántico de sistemas atómicos y moleculares está basado en las soluciones posibles de la ecuación de Schrödinger independiente del tiempo para sistemas compuestos por un electrón y un núcleo que interactúan según la ley de Coulomb. Estas soluciones son funciones de onda que representan los estados estacionarios admisibles para estos sistemas. El sistema más básico al cual se aplica la ecuación de Schrödinger, y que cuenta con solución exacta, es el átomo de hidrógeno que consta de un electrón y un protón. De todos modos, este no es el único sistema que puede resolverse de un modo exacto y analítico. De manera análoga se tratan los iones $\mathrm{He}^{+}, \mathrm{Li}^{2+}, \mathrm{Be}^{3+}$. Todos estos sistemas son denominados hidrogenoides pues, aunque difieren en la cantidad de protones en el núcleo, sólo contienen un electrón. En cambio, para aquellos átomos con más de un electrón, las diferentes soluciones de la ecuación de Schrödinger sólo se pueden obtener de manera aproximada debido a la dificultad de cómputo que introducen las interacciones inter-electrónicas. Si bien son muy 
diversos los métodos aproximados ${ }^{3}$ de los cuales se nutre la química cuántica, las aproximaciones que se usan en la práctica son de dos tipos. Por un lado, los métodos aproximados, denominados enlace de valencia y Orbital Molecular, se utilizan para encontrar la mejor forma de la función de onda que representa a un sistema molecular. Por otro lado, la aproximación Born-Oppenheimer permite sortear las dificultades de cómputo asociadas a la ecuación de Schrödinger de muchos cuerpos.

El análisis de estos métodos aproximativos nos ayudará a pensar la química cuántica como una disciplina que no se desarrolla exclusivamente a partir del formalismo mecánico cuántico, sino más bien a partir de modelos que se caracterizan por adquirir cierta autonomía respecto de la teoría cuántica.

\subsection{Enlace de valencia y orbital molecular}

Sobre la base del trabajo de Heitler y London, la química cuántica se encargó de la postulación de modelos con ciertas funciones de onda de prueba (trial wavefunctions) sobre las cuales se aplica el análisis variacional. La elección de tales funciones de onda tiene una influencia directa en la calidad del resultado buscado. Sin embargo, las propias técnicas aproximativas imponen pocas restricciones externas a la forma de esas funciones de onda de prueba. Consecuentemente, cualquier tratamiento es un procedimiento de búsqueda que comienza con una gran cantidad de posibilidades. Debido a que los resultados de estas técnicas son sólidos sólo cuando el conjunto de funciones de onda de prueba incluye algunas funciones que son aproximaciones razonables a la función de onda real, la selección de las funciones de onda de prueba constituye el mayor obstáculo para el tratamiento mecánico-cuántico de moléculas (Woody 2012).

La química cuántica desarrolló dos métodos de búsqueda de esas funciones de onda de prueba para sistemas moleculares conocidos como Enlace de Valencia (EV) y Orbital Molecular (OM). Cada uno de estos métodos incorpora un algoritmo particular para generar funciones de onda, y la justificación para elegir ese algoritmo descansa en supuestos conceptuales y cualitativos acerca de la forma que debería tener la función de onda molecular. Para EV, las moléculas están compuestas por sus átomos constituyentes, en los cuales los electrones tienen una ubicación localizada y se pueden individualizar asociándolos a un núcleo particular. De este modo, las funciones de onda deberán reflejar esta característica a través de una combinación lineal de funciones de onda atómicas correspondientes a los átomos dentro de la molécula. En este sentido EV es una extensión de los lineamientos desarrollados por Heitler y London.

Por su parte, $\mathrm{OM}$ concibe al sistema molecular como una nueva entidad en la cual los átomos constituyentes ya no pueden ser reidentificados y, por tanto, los electrones ya no pueden ser ubicados en torno a un único núcleo. Los electrones se encuentran deslocalizados en la molécula completa, de modo que las funciones de onda que describen este sistema incluyen componentes que asocian los mismos electrones a más de un núcleo. En otras palabras, para $\mathrm{OM}$ las moléculas no están compuestas por átomos, sino que, en tanto moléculas, son un todo inanalizable en componentes atómicos.

Es interesante resaltar la diferencia entre ambas imágenes: mientras que en EV los electrones ocupan orbitales atómicos, en OM los electrones ocupan orbitales moleculares. Mientras que en EV los orbitales atómicos se combinan a través del producto de las funciones de onda que los representan, en OM son los orbitales moleculares los que se combinan a través del producto de las funciones de onda que los representan. Por lo tanto, no se trata de modelos que brindan un mismo estado cuántico molecular pero expresado en distintas bases; por el contrario, EV y OM son diferentes modos de concebir la molécula, que dan lugar a diferentes estados cuánticos. Esto explica que, en muchos casos, ambos enfoques brinden diferentes resultados, como los que se refieren al carácter paramagnético del oxígeno, correctamente explicado por OM pero no por EV. Sin embargo, y a pesar de sus diferencias,

\footnotetext{
${ }^{3}$ Por ejemplo, cuando estas interacciones son dejadas de lado, los electrones pueden tratarse de forma independiente en términos de una función hidrogenoide, para después introducir los efectos promedio de las interacciones entre electrones mediante el método de HartreeFock.
} 
tanto EV como OM son modelos destinados a permitir la descripción de las propiedades y el comportamiento de sistemas moleculares.

Lo que nos resulta relevante destacar para el propósito del presente trabajo es que tales modelos incorporan elementos tanto de la mecánica cuántica como del ámbito clásico de la química estructural. En este sentido, no pueden ser considerados como modelos exclusivos de ninguna de ambas disciplinas. Sin duda, EV y OM no se encuentran a la misma "distancia" teórica de la mecánica cuántica y de la química estructural. Si bien EV incorpora la ecuación de Schrödinger independiente del tiempo, es un modelo más cercano a la manera estructural de pensar los problemas químicos, puesto que retiene la idea de una molécula compuesta de átomos. Por su parte, OM se aproxima más al tratamiento que brinda la mecánica cuántica de los sistemas compuestos, ya que la identidad de los componentes se desvanece al entrar en interacción. No obstante, ello no implica que se trate de un modelo estrictamente mecánico-cuántico porque, como señalaremos en el siguiente apartado, el supuesto de núcleo fijo que incorpora es incompatible con uno de los principios básicos de la teoría.

\subsection{La aproximación Born-Oppenheimer}

Un elemento central en el uso de la mecánica cuántica en química molecular es la aproximación BornOppenheimer (ABO) (Born \& Oppenheimer 1927).

Utilizando los subíndices $\alpha, \beta, \ldots$ para designar a los núcleos y los subíndices $i, j, \ldots$ para designar a los electrones, y llamando $\mathrm{Z}$ al número atómico de cada átomo, el Hamiltoniano total de una molécula genérica tiene la siguiente forma:

$$
H_{\text {total }}=\sum_{\alpha} \frac{P_{\alpha}^{2}}{2 M_{\alpha}}+e^{2} \sum_{\alpha<\beta} \frac{Z_{\alpha} Z_{\beta}}{\left|R_{\alpha}-R_{\beta}\right|}+\sum_{i} \frac{P_{i}^{2}}{2 M_{e}}-e^{2} \sum_{i} \sum_{\alpha} \frac{Z_{\alpha}}{\left|r_{i}-R_{\beta}\right|}+e^{2} \sum_{i<j} \frac{1}{\left|r_{i}-r_{j}\right|}
$$

donde el primer término representa la energía cinética $T_{n}$ de los núcleos, el segundo, la energía potencial $V_{n n}$ debida a la interacción entre los núcleos, el tercero, la energía cinética $T_{e}$ de los electrones, el cuarto, la energía potencial $V_{n e}$ debida a la interacción entre los núcleos y los electrones, y el último, la energía potencial $V_{e e}$ debida a la interacción de los electrones entre sí. A diferencia del caso de los sistemas hidrogenoides, este tipo de ecuación carece de solución analítica; por lo tanto, su tratamiento exige soluciones aproximadas.

La estrategia de la $\mathrm{ABO}$ se basa en la denominada aproximación de núcleos fijos: la interacción núcleo-electrón, representada por $V_{n e}\left(r_{i}, r_{\alpha}\right)$, se piensa en términos de electrones que se mueven alrededor de un núcleo fijo con una posición definida. Gracias a esta estrategia, el término de la energía cinética nuclear se desprecia. Esto significa que el primer término $T_{n}\left(P_{\alpha}\right)$ desaparece del Hamiltoniano. De este modo, se obtiene un Hamiltoniano electrónico de la siguiente forma:

$$
H_{e}=e^{2} \sum_{\alpha<\beta} \frac{Z_{\alpha} Z_{\beta}}{\left|R_{\alpha}-R_{\beta}\right|}+\sum_{i} \frac{P_{i}^{2}}{2 m_{e}}-e^{2} \sum_{i} \sum_{\alpha} \frac{Z_{\alpha}}{\left|r_{i}-R_{\beta}\right|}+e^{2} \sum_{i<j} \frac{1}{\left|r_{i}-r_{j}\right|}
$$

donde ahora se suponen los núcleos fijos en el espacio. Esto significa que las distancias internucleares $\left|r_{\alpha}-r_{\beta}\right|$ ya no se representan mediante operadores (diferencia entre los operadores de posición de los núcleos), sino como escalares: las posiciones nucleares son $r_{\alpha}$ representadas ahora como parámetros. Se trata de un Hamiltoniano electrónico porque los únicos operadores cuánticos corresponden a los electrones. Con este Hamiltoniano $H_{e}$, en lugar del $H_{\text {total }}$, se resuelve la ecuación de Schrödinger, y se obtienen los autoestados de la energía electrónica $E_{e}\left(r_{\alpha}\right)$ relativos al parámetro nuclear $r_{\alpha}$. Sólo en un segundo paso la energía cinética nuclear $T_{n}\left(p_{\alpha}\right)$ es introducida y la energía total $E_{\text {total }}$ se obtiene resolviendo la ecuación de Schrödinger independiente del tiempo para los núcleos. No obstante, aunque en diferentes posiciones espaciales, los núcleos siguen fijos en el espacio.

Es claro que la aproximación crucial de la $\mathrm{ABO}$ se introduce en el primer paso, donde las interacciones entre los electrones y los núcleos son tratadas en términos de electrones sometidos a un potencial coulombiano producido por núcleos fijos en posiciones definidas. Precisamente por ello, este 
paso de la $\mathrm{ABO}$ se conoce como aproximación de núcleo fijo (clamped-nucleus approximation), y constituye una estrategia básica en química cuántica para la descripción de la estructura molecular. En efecto, tal como se supone en la química estructural (no-cuántica), en la práctica el químico cuántico comienza por postular una estructura dada por las posiciones fijas de los núcleos en el espacio. Dicha estructura genera una "superficie" de energía potencial (PES por sus siglas en ingles) en el espacio de las fases del sistema, "sobre" la cual se calcula el comportamiento de los electrones. Los resultados de estos cálculos son los que finalmente se comparan con los resultados empíricos para conservar o descartar la estructura geométrica previamente supuesta. Tal como afirma Hasok Chang "asumiendo que el núcleo se encuentra fijo en el espacio en sus lugares 'clásicos' los químicos son capaces de usar la mecánica cuántica para calcular otros aspectos de moléculas tales como longitudes y energías de enlace precisas" (Chang 2015, p. 198).

La pregunta relevante es cómo se justifica la estrategia por la cual los núcleos son tratados como partículas clásicas en reposo en posiciones definidas, de modo que suministren el marco clásico que produce el campo eléctrico en el cual se mueven los electrones. Según la respuesta tradicional, la eliminación de $T_{n}\left(p_{\alpha}\right)$ se justifica asumiendo que $T_{n}>>T_{e}$, presunción que reside en el hecho de que la masa $M_{\alpha}$ de los núcleos es mucho más grande que la masa $m_{e}$ de los electrones: $M_{\alpha}>>m_{e}$. En particular, cuando el límite de las masas tiende a infinito, entonces $m_{e} / M_{\alpha} \rightarrow 0$ y $T_{n}\left(p_{\alpha}\right) \rightarrow 0$. No obstante, la pregunta crucial desde el punto de vista de la mecánica cuántica sería ¿de qué manera se justifica la inferencia de $M_{\alpha} \rightarrow \infty$ a $T_{n}\left(p_{\alpha}\right) \rightarrow 0$ ?

La primera estrategia proviene del ámbito de la mecánica clásica donde un cuerpo con masa infinita tiene energía cinética cero y, en consecuencia, tendrá una posición definida. Sin embargo, la descripción más completa de una molécula en química no se da en un ámbito clásico, sino que se produce dentro del contexto cuántico donde, precisamente, las intuiciones clásicas generalmente no funcionan.

Una respuesta más cercana al ámbito cuántico toma en cuenta que, si el Hamiltoniano electrónico $\mathrm{H}_{\mathrm{e}}$ no es una función del momento nuclear $\mathrm{p}_{\alpha}$, entonces conmuta con las posiciones nucleares $r_{\alpha},\left[H_{e}, r_{\alpha}\right]=0$. A su vez, en el límite $m_{e} / M_{\alpha} \rightarrow 0$ se da que $T_{n}\left(p_{\alpha}\right) \rightarrow 0$ y el Hamiltoniano electrónico se hace aproximadamente igual al Hamiltoniano total: $H_{\text {total }}=H_{e}\left(p_{i}, r_{i}, r_{\alpha}\right)$. En consecuencia, cuando $m_{e} / M_{\alpha} \rightarrow 0$, el Hamiltoniano total también conmuta con las posiciones nucleares $r_{\alpha},\left[H_{\text {total }}, r_{\alpha}\right]=0$. Sobre esta base, es posible explicar la posición fija de los núcleos: cuando una molécula está en un estado estacionario, su estado es un autoestado de $H_{\text {total }}$, y como consecuencia, tiene un valor definido del observable $H_{\text {total }}$. Como la molécula tiene un valor definido de su $H_{\text {total }}$, entonces $r_{\alpha}$, al conmutar con $H_{\text {total }}$, también tiene un valor definido. Esto significa que los núcleos están localizados en posiciones definidas. Aunque esta justificación parece más adecuada por pertenecer al ámbito de la cuántica, toma por hecho el valor definido de $H_{\text {total }}$. Sin embargo, esta suposición también es cuestionable desde el punto de vista cuántico, puesto que supone el vínculo autoestado-autovalor (para más detalles ver soluciones del átomo de hidrógeno en Lombardi y Castagnino 2010).

Como consecuencia de las dificultades teóricas que surgen para justificar la $\mathrm{ABO}$, creemos que es importante evaluar el tipo de límite que se usa en dicha aproximación. Intuimos que tal pregunta nos permitirá comprender mejor la dinámica procedimental de la química cuántica. Una posible categoría de análisis para acercarse a la $\mathrm{ABO}$ podría ser la distinción entre límites fácticos y contrafácticos propuesta por Bruer (1982) y Rohrlich (1989). Por ejemplo, la relación entre la relatividad especial y la mecánica clásica puede ser establecida por medio del límite fáctico $v / c \rightarrow 0$, esto es, una aproximación para velocidades (v) mucho menores que la velocidad de la luz (c). Dicha relación también se puede obtener cuando se hace tender la velocidad de la luz a infinito, $c \rightarrow \infty$. Sin embargo, este segundo límite es contrafáctico debido a que c tiene un valor fijo como constante universal. Otro caso de límite contrafáctico es el generado por el problema del límite clásico de la mecánica cuántica. Este límite clásico se obtiene a partir del límite fáctico $\hbar / S \rightarrow 0$ (que representa la situación en donde la acción $S$ es mucho más grande que el cuanto de acción $\hbar$ ), pero también puede obtenerse usando el límite contrafáctico $\hbar \rightarrow 0$. 
Entonces ¿qué tipo de límite es $m_{e} / M_{\alpha} \rightarrow 0$ de la $\mathrm{ABO}$ ? El límite $m_{e} \rightarrow 0$ es contrafáctico porque la masa del electrón es una constante. El límite $M_{\alpha} \rightarrow \infty$ también es un contrafáctico porque las masas de los núcleos de cualquier elemento químico también son constantes. Por lo tanto, el límite $m_{e} /$ $M_{\alpha} \rightarrow 0$ debe ser pensado como un límite práctico que expresa la gran diferencia entre las masas de los núcleos y los electrones, pero que no permite afirmar estrictamente que el Hamiltoniano total conmuta con las posiciones nucleares $r_{\alpha},\left[H_{\text {total }}, r_{\alpha}\right]=0$.

Sin embargo, la diferencia entre limite fáctico y límite contrafáctico no tiene en cuenta las consecuencias de la aproximación, y en este sentido no contempla un aspecto propio y fundamental de la $\mathrm{ABO}$. La distinción que establece tal límite es entre lo fácticamente posible y los fácticamente imposible. Pero para abordar la $\mathrm{ABO}$ es necesario establecer una distinción que tome en consideración si la aproximación tiene consecuencias consistentes o inconsistentes con la teoría que se intenta aplicar. Por ejemplo, en el caso de la mecánica clásica se puede suponer que la fricción de un cuerpo en movimiento en una superficie es cero. Este supuesto es probablemente falso debido a que no existe en el mundo real una superficie perfectamente lisa. Sin embargo, la idea que subyace a esta aproximación es compatible con la mecánica clásica: si bien una superficie con fricción cero no existe en la realidad, su existencia no contradice los postulados de la teoría ni sus consecuencias lógicas. La situación es completamente diferente si se supone, en el contexto de la relatividad especial, que dos objetos espacialmente separados interactúan instantáneamente: este supuesto contradice uno de los postulados de la teoría, en particular, el que fija a la velocidad de la luz (c) como la velocidad limite de cualquier interacción física.

Como mencionamos anteriormente, al considerar que las posiciones $\mathrm{r} \alpha$ de los núcleos conmutan con el Hamiltoniano $H_{\text {total }}$, la $\mathrm{ABO}$ supone al núcleo en una posición definida. Por otro lado, $T_{n}\left(p_{\alpha}\right) \rightarrow 0$ nos indica que el núcleo se encuentra en reposo. Con estos elementos puede resolverse la ecuación de Schrödinger, y se obtienen los estados energéticos de los electrones en una molécula. De este modo, la estructura química molecular queda definida en términos cuánticos, pero al costo de contradecir el Principio de Indeterminación de Heisenberg. En efecto, según este principio una partícula cuántica no puede tener de manera simultánea valores definidos de posición y momento, tal como supone ineludiblemente la $\mathrm{ABO}$ en relación a los núcleos. Como afirma Hasok Chang:

En esta aproximación de "fijación", los núcleos atómicos son considerados esencialmente como partículas clásicas; [...] esta imagen es no-cuántica de un modo muy fundamental, ya que la asignación simultánea de posición fija y momento fijo (esto es, cero) a tales partículas viola el principio de incertidumbre de Heisenberg. (Chang 2015, p. 198)

\section{Cómo operan los modelos en química cuántica}

Para entender las novedades epistemológicas que la química cuántica genera, analicemos sucintamente el modo en que se suele caracterizar el conocimiento científico desde la filosofía de la ciencia tradicional, a fin de evaluar si ello condice con la práctica del químico cuántico.

Según las perspectivas tradicionales imperantes durante el siglo XX, las teorías se constituyen como principales articuladores del conocimiento científico. Ello no significaba necesariamente relegar los modelos a funciones puramente propedéuticas o didácticas. Sin embargo, en tanto que la concepción semántica afirma que una teoría se presenta a partir de sus modelos (van Fraassen 1989, Díez \& Moulines 1997), precisamente delimitando y reglamentando mediante las leyes de la teoría las relaciones que pueden instituirse en el marco de un modelo, parece imponerse, desde tal perspectiva, una suerte de dependencia de los modelos respecto de la teoría. Es cierto que la dependencia no debe ser caracterizada como una relación de deducibilidad, puesto que ningún modelo se deduce estrictamente de las leyes fundamentales de una teoría sin que medie algún tipo de rectificación; e inclusive, en algunos casos, ni siquiera en tales instancias. De todos modos, y a pesar de ello, el requisito de dependencia sigue vigente en tanto que lo que se exige como condición mínima es que el modelo esté legitimado por la teoría, es decir, que las entidades del modelo se comporten tal y como 
las leyes lo prescriben. Tal como afirman Mauricio Suárez y Nancy Cartwright (2008) recordando las tesis de Ernan McMullin (1985),

\begin{abstract}
una actitud realista respecto de la teoría no necesita exigir que el modelo del fenómeno sea una consecuencia deductiva de la teoría, pero debe requerir que las simplificaciones introducidas en la descripción sean legitimadas por la teoría o por una descripción de los fenómenos que resultara aceptable [...]. Si así no fuera, la garantía no se trasladaría desde [la evidencia en favor de] el fenómeno a la teoría (Suárez \& Cartwright 2008, p. 67).
\end{abstract}

En efecto, para aquellas versiones confirmacionistas de la concepción semántica, que pretenden atribuir valores de verdad o grados de confirmación a las teorías, la dependencia modelo-teoría se vuelve un requisito ineludible. La interdependencia pretendida entre la teoría y el mundo supone una primigenia interdependencia entre la teoría y aquello con lo cual la teoría se aplica al mundo: el conjunto de sus modelos. Más precisamente, según esta concepción, entre el conjunto de los modelos posibles de una teoría existe un subgrupo que son los modelos pretendidos; y, si el mundo se encontrara en algunos de esos modelos, entonces la teoría quedaría derivativamente confirmada. En este sentido, Adams afirma que una "teoría es verdadera si y sólo si todos sus modelos pretendidos satisfacen sus axiomas, en caso contrario es falsa" (Adams 1959, p. 260). Por lo tanto, en el marco de la concepción semántica se considera que los modelos deben ser modelos de las teorías ya que en definitiva son sus «hacedores de verdad». Es cierto que las teorías, en tanto que no se identifican con entidades lingüísticas (tal y como lo suponían los partidarios de la concepción heredada), sino con entidades modelísticas expresadas bajo un sistema conjuntista, no pueden ser, estrictamente hablando, en sí mismas ni verdaderas ni falsas. Sin embargo, la noción de aserción empírica, introducida por los semanticistas, expresa, mediante un enunciado susceptible de ser verdadero o falso, la relación entre los sistemas empíricos de los que se pretende dar cuenta y los modelos determinados por la teoría. En este sentido, no sería erróneo afirmar, junto con José Díez y C. Ulises Moulines (1997, p. 339), que las teorías sí pueden ser derivativamente verdaderas.

Existen, es cierto, diferentes versiones de la concepción semántica. Sin embargo, la que actualmente tiene mayor vigencia es la concepción estructuralista (Sneed 1979, Stegmüller 1981). Según esta concepción, el mejor modo de reconstruir las teorías sería mediante la noción de red teórica: una red teórica es lo que se produce como consecuencia de una serie de especializaciones de las leyes fundamentales de una teoría. De este modo, la teoría no se identificaría sólo con las leyes fundamentales sino con el conjunto de las leyes fundamentales y las leyes especiales. La noción de especialización presupone la adición de ciertas constricciones adicionales a la ley fundamental para generar ciertas leyes especiales que den cuenta de diferentes situaciones experimentales. Por ejemplo, a partir de la ley fundamental de la mecánica clásica, $\bar{F}=m \bar{a}$, y producto de ciertas constricciones, se generan las leyes especiales que logran explicar las oscilaciones del péndulo o el movimiento sobre un plano inclinado. Definida entonces la teoría como un conjunto de modelos relacionados entre sí, la red teórica suele interpretarse como una estructura de tipo arbórea que se genera a partir de las sucesivas especializaciones.

Es en este contexto en donde cobra cierta relevancia analizar la utilización de modelos en química cuántica. En primer lugar, debe señalarse la convivencia, en el seno de la misma disciplina, de dos enfoques que se consideran rivales. Si bien en principio los modelos EV y OM brindan diferentes resultados, en muchos casos ambos pueden aplicarse con éxito a un mismo sistema: en estos casos los resultados tienden a converger. No obstante, esto no cancela la diferencia conceptual entre ambos: "La equivalencia en el límite teórico no implica que los diferentes modos en que las teorías del enlace de valencia y del orbital molecular simplifican la función de onda multielectrónica conducirían a explicaciones equivalentes de, por ejemplo, el benceno" (Hendry 2004, p. 1057). La diferencia entre los dos modelos no reside, entonces, en los aspectos teóricos de ambos, sino en el modo en que EV y OM describen la estructura interna de la molécula: mientras que para EV la molécula es una entidad compuesta donde es posible continuar identificando los átomos componentes, para OM la molécula es un todo inanalizable en componentes atómicos. Pero en lugar de preocuparse por resolver esta 
incompatibilidad, los químicos cuánticos conviven con ella: su utilización no depende de que brinden una descripción teórica coherente de un dominio que constituye su referente, sino que se los utiliza sobre la base de su éxito práctico.

Por otra parte, la química cuántica, incluso en la formulación de los modelos EV y OM, se basa en la introducción de la $\mathrm{ABO}$ : el pasaje desde el Hamiltoniano exacto al Hamiltoniano electrónico funda las bases de la química cuántica, en tanto que dicha aproximación está en el núcleo duro de esta disciplina. Efectivamente es gracias a ella que los químicos lograrían recuperar la tan preciada estructura molecular que les permite generar el grueso de la información respecto de las sustancias.

Tal como fue señalado, la peculiaridad de la $\mathrm{ABO}$ se debe a que integra de un modo constructivo dos teorías incompatibles: introduce, por un lado, la ecuación de Schrödinger propia de la mecánica cuántica; pero, por otra parte, para permitir resolver la ecuación en cuestión en sistemas polinucleares, anula el movimiento del núcleo presuponiéndolo como una partícula clásica. Es cierto que este límite práctico es una simplificación matemática para hacer más tratable al sistema ante la imposibilidad de resolver las ecuaciones de un modo analítico y exacto; pero este caso presenta una característica peculiar. Si bien este tipo de estrategias suele usarse a menudo sin mayores inconvenientes (pensemos, por ejemplo, cuando se supone un plano si fricción o mejor aún cuando surge el denominado problema de los tres cuerpos), lo que se torna imperioso resaltar es que, en el caso particular de la $\mathrm{ABO}$, dicha simplificación matemática genera una seria dificultad conceptual. Como afirma Hasok Chang:

podría decirse que la mecánica cuántica de Schrödinger, ya desde su primer uso para un sistema de la vida real, nació con el supuesto del núcleo fijo. Debe enfatizarse nuevamente que esto no es algo que surge por la necesidad de aproximación, sino algo entretejido en la propia trama de la teoría cuántica elemental. El marco teórico de la mecánica ondulatoria de Schrödinger no brinda margen alguno para teorizar acerca del estado del núcleo (Chang 2015, p. 199).

Pero el supuesto del núcleo fijo entra en contradicción con uno de los principios básicos de la mecánica cuántica. Precisamente es esta dificultad la que nos obliga a repensar, por un lado, la estructura interna de la química cuántica como disciplina y, por el otro, el tipo de teoría cuántica de la que hace uso la química cuántica.

Respecto del primer punto, puede afirmarse que en el contexto de la química cuántica los modelos adquieren independencia y autonomía respecto de la teoría. En primer término, el éxito pragmático del modelo no puede traducirse, tal y como suponen algunos partidarios de la concepción semántica (en especial aquellos que intentan brindar algún tipo de perspectiva realista), como un elemento en favor de la verdad de la teoría. En efecto, en tanto que el modelo involucra dos teorías (química estructural y mecánica cuántica) que resultan incompatibles (es decir, que como tales no pueden ser ambas verdaderas), en química cuántica no tiene sentido pensar los modelos como hacedores de verdad de una teoría. En efecto, los modelos no se instituyen como subsidiarios y dependientes de la teoría, sino que, por el contrario, adquieren un grado de libertad que pone en evidencia la prioridad de los modelos por sobre las teorías: los modelos toman de las teorías sólo aquello que les resulta útil para dar cuenta del fenómeno a explicar. En química cuántica, el modelo no es una mera instancia útil para la aplicación de la teoría, sino que, por el contrario, las propias teorías se convierten en herramientas útiles para la construcción de los modelos.

A su vez, el caso de los modelos en química cuántica no es susceptible de ser analizado en términos de una estructura arbórea, al modo que suele pensarse desde una perspectiva estructuralista. Los modelos basados en el Hamiltoniano electrónico no se formulan como una especialización, como un caso particular donde la teoría puede aplicarse. Es decir, dichos modelos no parecen ser ni un caso particular de la mecánica cuántica, ni un caso particular de la física clásica encarnada en la química molecular. Nuevamente, en tanto que involucran teorías incompatibles, los modelos de la química cuántica cobran autonomía respecto de ellas y, por lo tanto, no pueden interpretarse como siendo parte de una red teórica específica. 
Respecto al segundo punto, esto es, qué tan cuántica es la química cuántica, puede afirmarse que es una disciplina "intermedia". En términos de Gavroglu \& Simões (2015), se trata de un campo intermediario ("in-between"), cuyas bases no deben concebirse como teorías físicas "incompletas" o esquemas "preteóricos" a ser abandonados con el desarrollo de la física: la química cuántica cuenta con sus propios recursos que continuarán evolucionando con el desarrollo de la disciplina. Incluso, no sería aventurado afirmar que, a pesar de que su formalismo es estrictamente cuántico, es una disciplina clásica debido a que la química, bajo ningún aspecto, puede desentenderse de la noción de estructura molecular. En efecto, es dicha noción la que le permite desarrollar todo el cuerpo de conocimiento químico acerca de las sustancias. Como afirma Robin Hendry, "la estructura molecular es tan central para la explicación química, que explicar la estructura molecular es prácticamente como explicar la química completa” (Hendry 2010, p. 183). Pero la noción de estructura es netamente clásica, ya que para su determinación es necesario definir los valores de posición y momento para todos sus elementos, esto es, los núcleos de los átomos que componen la molécula.

Quizás pueda pensarse que el problema aquí presentado es meramente contingente en tanto que su resolución depende simplemente de ciertos avances computacionales que permitan solucionar el Hamiltoniano total para los sistemas abiertos. Sin embargo, esta opinión se enfrenta al menos a dos contraargumentos. En primer lugar, desde un punto de vista epistemológico, el interés del presente trabajo es comprender la química cuántica tal como ella se desenvuelve en la actualidad. Adscribir un carácter provisorio y superable al modo en que se recurre a teorías incompatibles en química cuántica, si bien puede ser una tesis que merece ser explorada en el marco de la filosofía de la ciencia, es lógicamente independiente a la discusión sobre el tipo de cuántica que se utiliza actualmente en la práctica de la disciplina. En segundo lugar, y fundamentalmente, existen casos, como el de los isómeros ópticos, donde la posibilidad de obtener el Hamiltoniano total no permite develar la diferencia estructural entre ellos (ver Lombardi, Fortin \& Martínez González 2016). A su vez, puesto que la ecuación de Schrödinger determina la energía del sistema, no es de esperar que sus soluciones puedan determinar ni confirmar estructura geométrica alguna. Esto se debe a que una de las peculiaridades del formalismo cuántico consiste en no poder determinar simultáneamente el valor de observables que no conmutan, en particular de la posición y el momento. En este sentido la introducción de ciertos elementos estructurales clásicos en los sistemas cuánticos aislados, tal como lo hace la $\mathrm{ABO}$ cuando elimina el término de la energía cinética del núcleo, resulta esencial para la disciplina; y, consecuentemente, no constituye una estrategia a ser superada con el avance de la ciencia.

\section{Conclusiones}

En el presente trabajo hemos discutido las peculiaridades del modo en que se ha desarrollado la química cuántica desde su surgimiento a principios del siglo XX. Para ello, en primer lugar, hemos señalado la necesidad de introducir, en el ámbito de la química, el formalismo de la mecánica cuántica para dar cuenta de la energía de los sistemas químicos. Sin embargo, como explicamos, el tránsito de la química estructural clásica a la química cuántica genera una serie de dificultades conceptuales en relación al modo en que se debe comprender el desarrollo reciente de las ciencias químicas. En efecto, la consideración de los modelos de Enlace de Valencia y de Orbital Molecular, así como también el análisis de la aproximación Born-Oppenheimer, han puesto en evidencia que los modelos en química cuántica tienen la peculiaridad de instituirse de un modo autónomo en relación al cuerpo teórico, ya que todos los casos incorporan dos marcos teóricos incompatibles: la física clásica en su aplicación a la química estructural y la mecánica cuántica.

Asimismo, si se admite que la química no puede desentenderse de la noción de estructura molecular, y siendo ésta una noción eminentemente clásica (ya que para definir la estructura es necesario determinar la posición y el momento de todos sus elementos), parece ineludible concluir que la clasicidad de la química cuántica no es una mera cuestión contingente por superar con el futuro desarrollo de nuevas técnicas computacionales. En la medida en que sea necesario mantener la noción 
de estructura molecular para dar cuenta de los comportamientos de las sustancias, el carácter híbrido que actualmente posee la química cuántica resultará ser un rasgo característico de ella.

Esta peculiaridad de la química cuántica nos permitió, a su vez, delinear una noción de modelo diferente de la usual. Los modelos en química cuántica ni son dependientes ni actúan como "hacedores de verdad" de las teorías. Por el contrario, los modelos en química cuántica adquieren su legitimidad exclusivamente en función al éxito pragmático: incorporan de las diferentes teorías físicas aquello que sea útil para los propósitos de la investigación, sin importar si ello supone la aceptación simultánea de marcos teóricos incompatibles.

Bibliografía

Adams, E. W. (1959), "The Foundations of Rigid Body Mechanics and the Derivation of Its Laws from Those of Particle Mechanics”, en Henkin, L., Suppes, P. y A. Tarski (eds.), The Axiomatic Method, Amsterdam: North Holland, pp. 250-265.

Born, M. y J. Oppenheimer (1927), “Zur Quantentheorie der Molekeln”, Annalen der Physik 84: 457-484.

Bruer, J. T. (1982), "The Classical Limit of Quantum Theory", Synthese 50: 167-212.

Chang, H. (2015), "Reductionism and the Relation Between Chemistry and Physics" en Arabatzis, T., Renn, J. y A. Simões (eds.), Relocating the History of Science: Essays in Honor of Kostas Gavroglu, New York: Springer, pp. 193-209.

Díez, J. A. y C. U. Moulines (1997), Fundamentos de Filosofia de la Ciencia, Barcelona: Ariel Editorial.

Gavroglu, K. y A. Simões, (2012), Neither Physics nor Chemistry: A History of Quantum Chemistry, Cambridge, MA: MIT Press.

Gavroglu, K. y A. Simões (2015), "Philosophical Issues in (Sub)Disciplinary Contexts. The Case of Quantum Chemistry", en Scerri, E. y G. Fisher (eds.), Essays in the Philosophy of Chemistry, Oxford: Oxford University Press, pp. 60-79.

Heitler, W. y F. London (1927), "Wechselwirkung neutraler Atome und homöpolare Bindung nach der Quantenmechanik", Zeitschrift für Physik 44: 455-472.

Hendry, R. F. (2010), "Ontological Reduction and Molecular Structure", Studies in History and Philosophy of Modern Physics 41: 183-191.

Knuuttila, T. (2005), "Models, Representation and Mediation”, Philosophy of Science 72: 1260-1271.

Kragh, H. (1977), "Chemical Aspects of Bohr's 1913 Theory", Journal of Chemical Education 54: 208-210.

Lewis, G. N. (1916), “The Atom and the Molecule”, Journal of the American Chemical Society 38: 762-785.

Lombardi, O. y M. Castagnino (2010), "Matters Are Not So Clear on the Physical Side", Foundations of Chemistry 12: 159-166.

Lombardi, O., Fortin, S. y J. C. Martínez González (2016), "Isomers and Decoherence", Foundations of Chemistry 18: 225-249.

McMullin, E. (1985), "Galilean Idealization", Studies in History and Philosophy of Science 16: 247-26.

Morgan, M. (1999), "Learning from Models", en Morgan, M. y M. Morrison (eds.), Models as Mediators, Cambridge: Cambridge University Press.

Morgan, M. y M. Morrison (1999), "Models as Mediating Instruments", en Morgan, M. y M. Morrison (eds.), Models as Mediators, Cambridge: Cambridge University Press, pp. 10-37.

Morrison, M. (2015), Reconstructing Reality: Models, Mathematics, and Simulations, Oxford: Oxford University Press.

Parson, A. L. (1915), "A Magneton Theory of the Structure of the Atom”, Smithsonian Miscellaneous Collections 65: 1-80.

Rohrlich, F. (1989), “The Logic of Reduction: The Case of Gravitation”, Foundations of Physics 19: 1151-1170.

Sneed, J. D. (1971), The Logical Structure of Mathematical Physics, Dordrecht: Reidel, 2a ed. revisada, 1979. 
Stegmüller, W. (1981), La concepción estructuralista de las teorías, Madrid: Alianza.

Suárez, M. (1999), "The Role of Models in the Application of Scientific Theories: Epistemological Implications", en Morgan, M. y M. Morrison (eds.), Models as Mediators, Cambridge: Cambridge University Press, pp. 168-196.

Suárez, M. y N. Cartwright (2008), "Theories: Tools versus Models", Studies in History and Philosophy of Modern Physics 39: 62-81.

Woody, A. I. (2012), "Concept Amalgamation and Representation in Quantum Chemistry" en Woody, A. I., Hendry, R. F. y P. Needham (eds.), A Handbook of Philosophy of Science, vol. 6: Philosophy of Chemistry, Oxford: Elsevier, pp. $427-466$. 